\title{
A CFD study on wall shear stress of falling water film on food contact surface
}

\author{
Nawaporn Suthanarak ${ }^{1}$, and Navaphattra Nunak $^{1, *}$ \\ ${ }^{1}$ Food Engineering Department, Faculty of Engineering, King Mongkut's Institute of Technology Ladkrabang, Bangkok, Thailand
}

\begin{abstract}
This paper proposes a CFD model to simulate the wall shear stress of the falling water film on the hydrophilic surface (aluminum) of the inclined flat plate in the range of $30^{\circ}, 60^{\circ}$ and $90^{\circ}$ at a flow rate of 100,200 and $300 \mathrm{~L} / \mathrm{h}$. Wall shear stress simulated from the CFD using SST k- $\omega$ with the VOF model was compared with the real data obtained from the experiment. It was found that both inclined angle and water flow rate had an effect to the wall shear stress and the CFD with the proposed model had the potential to predict it with the maximum averaged error of $2.9 \%$.
\end{abstract}

\section{Introduction}

In food industry, the removal of soil and microorganism from food processing equipment and machines in wetcleaning with water is the most important process indicating the level of food safety. If inadequate or improper cleaning occurs, soil residues may remain on the equipment surface causing to product contamination and deterioration and foodborne disease in the consumers.

Generally, cleaning of a large tank with the principle of falling film using a spray ball is preferable. A series of jets of water spraying injected through spray balls spread out on the interior surface of a tank and flowed down by gravity force creating the wall shear stress for removing soil from the surface. From a theory of fluid mechanics, the wall shear stress is an important factor to indicate the cleaning performance. It depends on many parameters such as liquid properties, flow characteristics and inclination of the surface [1-4]. Fuchs et al. [3] and Lerch et al. [5] have studied the cleaning process with falling water film and found that the turbulent flow of water can enhance the cleaning performance. This finding also is in the agreement with Tamime [2]. Therefore, an understanding on flow characteristics of cleaning fluid effecting to cleaning performance in term of wall shear stress is importance.

To obtain the wall shear stress, it can be achieved by directly calculating from the measured thickness of liquid film. However, it spends a lot of time and costs a lot. Therefore, Computational Fluid Dynamics (CFD) is introduced to simulate the wall shear stress [6-9]. Moreover, it is also widely used to simulate the flow velocity of fluid and other applications about a decade [10-11]. In this study, the CFD model was proposed to estimate the wall shear stress of falling water film created on the inclined surface at various flow rates.

\section{Theory of falling liquid film}

Falling liquid film is a film flow downward under gravity, the characteristics of film flow can be indicated by film Reynolds number $\left(R e_{\mathrm{f}}\right)$ as illustrated in equation (1) $[3,12]$ :

$$
\operatorname{Re}_{\mathrm{f}}=\Gamma / \eta=\Gamma_{\mathrm{V}} / v=\dot{V} / B v
$$

where $\Gamma$ is the mass flow rate per wetted width $(\mathrm{kg} / \mathrm{h} \cdot \mathrm{m})$, $\eta$ is the dynamic viscosity of the liquid $(\mathrm{kg} / \mathrm{m} \cdot \mathrm{s}), \Gamma_{\mathrm{V}}$ is the volumetric wetting rate $\left(\mathrm{m}^{3} / \mathrm{h} \cdot \mathrm{m}\right), v$ is the kinematic viscosity of the liquid $\left(\mathrm{m}^{2} / \mathrm{s}\right), \dot{V}$ is the volume flow rate $\left(\mathrm{m}^{3} / \mathrm{h}\right)$ and $B$ is the wetted width $(\mathrm{m})$.

According to equation (1), the film flow characteristic can be classified into three types as follow: $R e_{\mathrm{f}}<4$ is laminar flow or smooth laminar film, $4<R e_{\mathrm{f}}<$ 400 is transient flow which is a laminar film with a small wave on the interface and $R e_{\mathrm{f}}>400$ is a turbulent flow or wavy film having a large wave on the interface (fig. 1) [12].

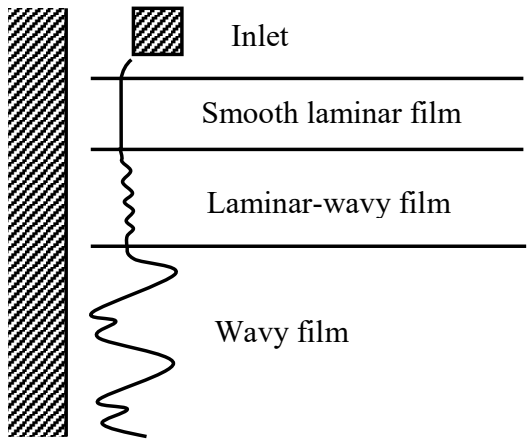

Fig. 1. Falling film characteristic [12]

The thickness of liquid film can be evaluated from the theory of Nusselt film which assumes that the film flow characteristic is laminar under the action of gravity,

\footnotetext{
Corresponding author: Navaphattra@gmail.com
} 
the shear stress at interface is neglected, and fluid properties are constant $[3,4,13]$. The film thickness $(\delta)$ can be calculated by equation (2)

$$
\delta=\left(3 v^{2} \operatorname{Re}_{\mathrm{f}} / \mathbf{g} \sin \theta\right)^{1 / 3}
$$

where $\mathbf{g}$ is the gravitational acceleration $\left(\mathrm{m} / \mathrm{s}^{2}\right)$ and $\theta$ is the angle of inclination of the surface with respect to a horizontal.

During the film flows down, there is a force acting upwards created between the surface and the liquid film that is called the wall shear stress, influenced by the liquid film thickness. It is an important parameter indicating the performance of soil removal from the surface. It can be calculated by equation (3) with the several assumptions, i.e. the fluid flow is uniform and incompressible, the net pressure force is zero, and the momentum flux through the control volume is zero [14].

$$
\boldsymbol{\tau}_{\mathbf{w}}=\rho \mathbf{g} \delta \sin \theta
$$

where $\tau_{\mathbf{w}}$ is the wall shear stress $(\mathrm{Pa}), \rho$ is density $\left(\mathrm{kg} / \mathrm{m}^{3}\right)$.

\section{Materials and methods}

\subsection{The experimental setup}

The experimental unit (see fig. 2) consisted of a centrifugal pump, a tube, a ball valve, a water container, and a testing surface. For the testing surface, an 10x50 $\mathrm{cm}$ aluminum plate used as representative of food contact surface, can be adjusted for any angle. Water was circulated by the centrifugal pump (Sonic AP1600 20-23 watt), installed in a water container with a tube and a ball valve connecting, from a water container to overflow on a testing surface.

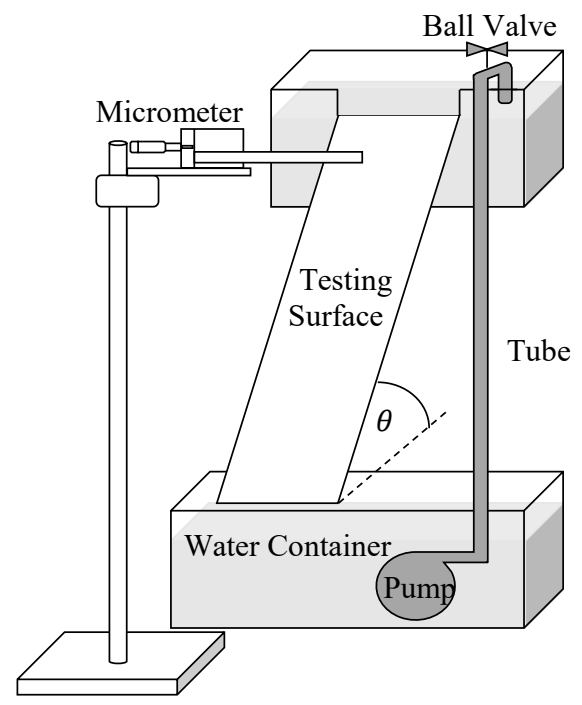

Fig. 2. Experimental Unit

\subsection{Experimental method}

The experiments were carried out under gravity driven falling water film on a vertical and inclined testing surface at various angles $\left(30^{\circ}, 60^{\circ}\right.$ and $\left.90^{\circ}\right)$. Water was circulated at least 5 minutes before the data were recorded. The flow rate of water was measured using weighing method (SF-400A resolution $0.1 \mathrm{~g}$ ) and the temperature of water was measured by thermocouple type K (Fluke 54IIB Thermometer S2). Experiments were performed at the flow rate of 100, 200 and $300 \mathrm{~L} / \mathrm{h}$ with Reynolds number of 311, 622 and 932, respectively. The micrometer with resolution of $0.01 \mathrm{~mm}$ was installed on the tripod to measure the thickness of water film at every $5 \mathrm{~cm}$ long from the origin point $(0$ $\mathrm{cm}$ ) (fig. 3), then compared with the theoretical results calculated from the equation (2) to consider the real data of water film thickness for calculating wall shear stress created on the surface by equation (3) $[3,4,13]$. They were referred as the real data for validating the results simulated from CFD.

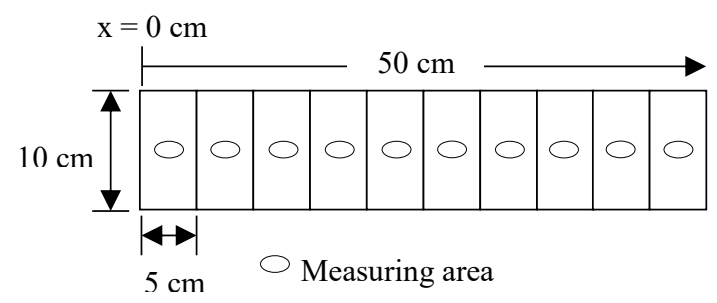

Fig. 3. Film thickness measuring position

\subsection{CFD Modeling}

In this study, the falling film flow was investigated by the three-dimensional CFD simulation (ANSYS FLUENT 16.0) with Navier-Stokes equation, which assumes that fluid is incompressible flow and constant viscosity. The shear stress transport $\mathrm{k}-\omega$ (SST k- $\omega$ ), a turbulent model, was used to simulate film flow with the sensitivity inside and outside shear layer [15]. From the preliminary experiments, it was found that the SST k- $\omega$ model gave the minimum error comparing with the wall shear stress simulated from other turbulent models, i.e. STD k- - , RNG k- $\varepsilon$ and Realizable k- $\varepsilon$ models (results not shown).

In addition, the multiphase model, Volume of Fluid (VOF) was also applied with SST k- $\omega$ model and hexagonal mesh for capturing the interface between two phases since the film flow in this study was two-phase flow (gas and liquid) [8, 15].

The wall shear stress of falling water film was simulated with the CFD model by separating the water film into many small elements, then it was considered under the conditions as shown in Table 1.

To validate the CFD model, the simulated wall shear stress was compared with the real data or theoretical data obtained from section 3.2 in term of a relative error as presented in equation (4)

$$
\% \text { error }=\left|\left(x_{\text {sim }}-x_{\mathrm{t}}\right) / x_{\mathrm{t}}\right| \mathrm{x} 100
$$

where \%error is a relative error, $x_{\text {sim }}$ is a simulation data and $x_{\mathrm{t}}$ is a real data. 
Table 1. Simulation conditions fixed in the CFD

\begin{tabular}{|c|c|}
\hline Parameter & Detail \\
\hline \multicolumn{2}{|l|}{ Operating Conditions } \\
\hline Gravity $\left(\mathrm{m} / \mathrm{s}^{2}\right)$ & 9.81 \\
\hline Pressure $(\mathrm{kPa})$ & 101.325 \\
\hline Surface Tension $(\mathrm{N} / \mathrm{m})$ & 0.072 \\
\hline \multicolumn{2}{|l|}{ Model } \\
\hline Viscous Model & SST k- $\omega$ \\
\hline Multiphase Model & VOF \\
\hline \multicolumn{2}{|l|}{ Phase } \\
\hline Primary Phase & $\operatorname{Air}\left(25^{\circ} \mathrm{C}\right)$ \\
\hline - Density $\left(\mathrm{kg} / \mathrm{m}^{3}\right)$ & $1.184^{\mathrm{a}}$ \\
\hline - Viscosity (kg/m-s) & $1.849 \times 10^{-5} \mathrm{a}$ \\
\hline Secondary Phase & Water $\left(25^{\circ} \mathrm{C}\right)$ \\
\hline - Density $\left(\mathrm{kg} / \mathrm{m}^{3}\right)$ & $997^{\mathrm{b}}$ \\
\hline - Viscosity (kg/m-s) & $0.000891^{\mathrm{b}}$ \\
\hline \multicolumn{2}{|l|}{ Boundary Conditions } \\
\hline Inlet water flow rate $(\mathrm{L} / \mathrm{h})$ & 100,200 and 300 \\
\hline
\end{tabular}

\section{Results and discussion}

\subsection{Wall shear stress validation}

Fig. 4 shows the thickness of water film calculated from the Nusselt's film theory and measured by a micrometer. The thickness measured from the experimental unit are in a good agreement with the findings of Fuchs et al. [3]. The thickness values obtained from both methods were very close for flow characteristic in the laminar or transient $(100 \mathrm{~L} / \mathrm{h})$, whereas the values obtained from the measurement were higher than that from the theory at higher flow rate $(200$ and $300 \mathrm{~L} / \mathrm{h}$ ) or in the turbulent flow. Since the Nusselt's film theory is scoped only for the laminar film flow, the calculated results are lower than the actual film thickness. Besides, cleaning the internal surface of a large tank should be processed with the turbulent falling film flow. Therefore, in this study, the wall shear stress considered to be the real data was calculated from the measured film thickness and used to validate the CFD model.

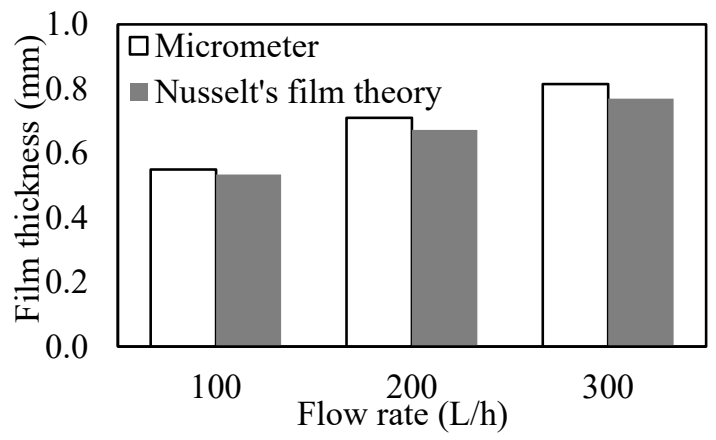

Fig. 4. Water film thickness on the inclined surface at $30^{\circ}$ calculated from Nusselt's film theory and measured by micrometer
Fig. 5 shows an example of comparing the wall shear stress obtained from the CFD simulation and the experiment at every $5 \mathrm{~cm}$ long from the origin point $(0 \mathrm{~cm})$. It was found that the CFD simulation had much relative error at the first one fifth of the whole distance due to the influence of the water inlet of the experimental unit. For this reason, the proposed CFD model could be used to simulate the film flow at the distance of $10-50 \mathrm{~cm}$ from the origin point. The contour of wall shear stress created on the inclined surface as shown in fig. 6. Therefore, the wall shear stress hereafter was the CFD averaged from the distance of 10 to $50 \mathrm{~cm}$ as presented in Table 2.

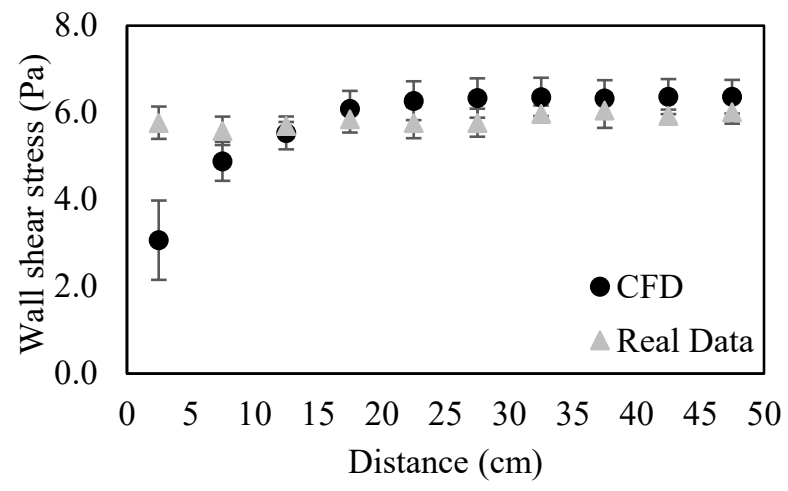

Fig. 5. Wall shear stress simulated from CFD and the real data obtained from the experiment on the inclined surface at $60^{\circ}$ with a flow rate of $300 \mathrm{~L} / \mathrm{h}$

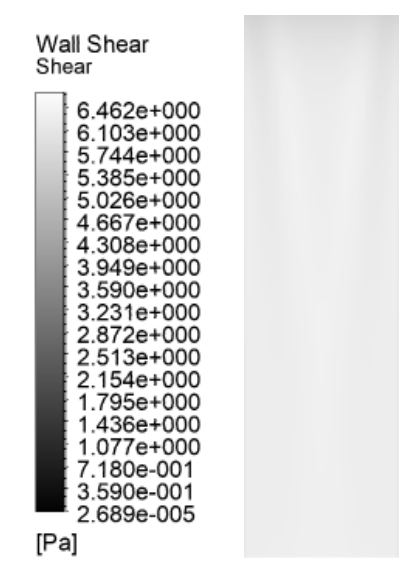

Fig. 6. Wall shear stress contour of water film flow on the inclined surface at $90^{\circ}$ with a flow rate of $200 \mathrm{~L} / \mathrm{h}$

Considering Table 2, It was found that the thickness of water film simulated from CFD had a good agreement with Fuchs et al. [3] and represented the low relative error. The maximum relative error under all experimental conditions was less than $3 \%$. This probably due to different assumptions of the CFD model and the equation (3). Since aluminum plate is a hydrophilic surface having the surface tension close to other types of food contact materials such as stainless steel, this model could also be applied to simulate the wall shear stress created on the food contact surface. The higher surface tension is, the higher wall shear stress is [6]. However, for more accuracy the further work on the correction factor for any types of materials would be needed. 
Table 2. Comparison of experimental calculation and CFD simulation with relative error

\begin{tabular}{|c|c|c|c|c|c|c|c|c|c|}
\hline \multirow{2}{*}{$\begin{array}{c}\text { Flow } \\
\text { rate } \\
(\mathrm{L} / \mathrm{h})\end{array}$} & \multicolumn{6}{|c|}{ Wall shear stress (Pa) with different inclined angles and relative error (\%) } \\
\cline { 2 - 11 } & \multicolumn{3}{|c|}{$30^{\circ}$} & \multicolumn{3}{c|}{$60^{\circ}$} & \multicolumn{5}{c|}{$90^{\circ}$} \\
\hline 100 & 2.689 & 2.624 & 2.437 & 3.879 & 3.767 & 2.895 & 4.323 & 4.198 & 2.900 \\
\hline 200 & 3.472 & 3.464 & 0.247 & 5.082 & 5.061 & 0.407 & 5.634 & 5.592 & 0.747 \\
\hline 300 & 3.986 & 3.941 & 1.112 & 5.844 & 5.761 & 1.432 & 6.367 & 6.385 & 0.283 \\
\hline
\end{tabular}

\subsection{Effect of inclined angle and flow rate}

From Table 2, wall shear stress of water film on the surface increased with the increasing of surface inclination and water flow rate as water film was influenced by gravity at a more inclined angle. When considering the water flow rate, the wall shear stress occurred form water film flow at 100 liters per hour, non-turbulent flow, was lower than that of at 200 and 300 liters per hour, turbulent flow. The higher water flow rate caused the film flow more turbulent, and more wall shear stress. However, the film wall shear stress is influenced by the water flow rate more than an inclined angle.

\section{Conclusions}

Wall shear stress is an important factor for cleaning by falling liquid film. It was influenced by water flow rate and inclined angle. The increasing of inclination angle and water flow rate caused the greater wall shear stress. When comparing wall shear stress simulated from the CFD using SST k- $\omega$ with VOF model and obtained from the experiments, it was found that the CFD was a potential tool to predict the wall shear stress at different conditions with the maximum error of $2.9 \%$. Nevertheless, further research would be needed in case of changing the material type. Finally, this study would be more benefits if this concept was applied to the cleaning behavior of any types of soils using falling water film in the future work.

The authors would like to thanks Mr. Kittisak Srichaitamrong and Ms.Natnaree Phromma for performing film thickness measurement.

\section{Reference}

1. K. R. Goode, K. Asteriadou, P. T. Robbins, P. J. Fryer, "Fouling and cleaning studies in the food and beverage industry classified by cleaning type," IFT, 12, 121-143, (2013)

2. Y. Tamime, Cleaning-in-place: Dairy, Food and beverage operations Third Edition, Blackwell Publishing, Oxford, (2008)

3. E. Fuchs, A. Boye, R. Murcek, J.-P. Majschak, "An experimental comparison of film flow parameters and cleaning behavior of falling liquid film for different tilt angles," Food Bioprod. Process., 93, 318-326, (2014)

4. A. To, S. R. Annapragada, "Experimental parameters for small-scale cleaning characterization. Part II: effect of fluid velocity on the kinetics of cleaning, Insitute of validation technology, (2015)

5. K. Lerch, J. Hinrichs, P. Dittmer, J. Rauschnabel, "Cleanability of surfaces from active pharmaceutical ingredient surrogate riboflavin by falling film," Chemie Ingenieur Technik, 85, 323332, (2013)

6. H. Lan, J. L. Wegener, B. F. Armaly, J. A. Drallmeier, "Developing laminar gravity-driven thin liquid film flow down an inclined plane," $J$. Fluids Eng., 132, (2010)

7. B. B. B. Jensen, A. Friis, Th. Benezech, P. Legentilhomme, C. Lelievre, "Local wall shear stress variations predicted by computational fluid dynamics for hygienic design," Food Bioprod. Process., 83(C1), 53-60, (2005)

8. Y. Xu, J. Yuan, J. U. Repke, G. Wozny, “CFD study on liquid flow behavior on inclined flat plate focusing on effect of flow rate," Eng. Appl. Comp. Fluid, 6, 186-194, (2012)

9. Y. $\mathrm{Yu}$, "A computational fluid study of falling film behavior on flat plate," FEDSM2014, (2014)

10. T. Norton, D.-W. Sun, "Computational fluid dynamics (CFD) - an effective and efficient design and analysis tool for the food industry: A review," Trends Food Sci. Technol., 17, 600-620, (2006)

11. S. Salo, A. Friis, G. Wirtanen, "Cleaning validation of fermentation tanks," Food Bioprod. Process., 86, 204-210, (2008)

12. F. Weise, S. Scholl, "Evaporation of pure liquids with increased viscosity in falling film evaporator," Heat Mass Transfer,45, 1037-1046, (2009)

13. S. Portalski, "Studies of falling liquid film flow: Film thickness on a smooth vertical plate," Chem Eng. Sci., 18, 787-804, (1963)

14. W. R. Fox, T. A. McDonald, Introduction to fluid mechanics, John Wiley \& Sons, New York, (1994)

15. ANSYS FLUENT, ANSYS FLUENT Theory guide, ANSYS Inc., (2011)

16. Y. A. Cengel, J. M. Cimbala, Fluid mechanics: fundamentals and applications, McGraw-Hill, New York, (2006) 